\title{
DYNA3D Code Practices and Developments
}

\author{
J. Lin, E. Zywicz, P. Raboin
}

U.S. Department of Energy

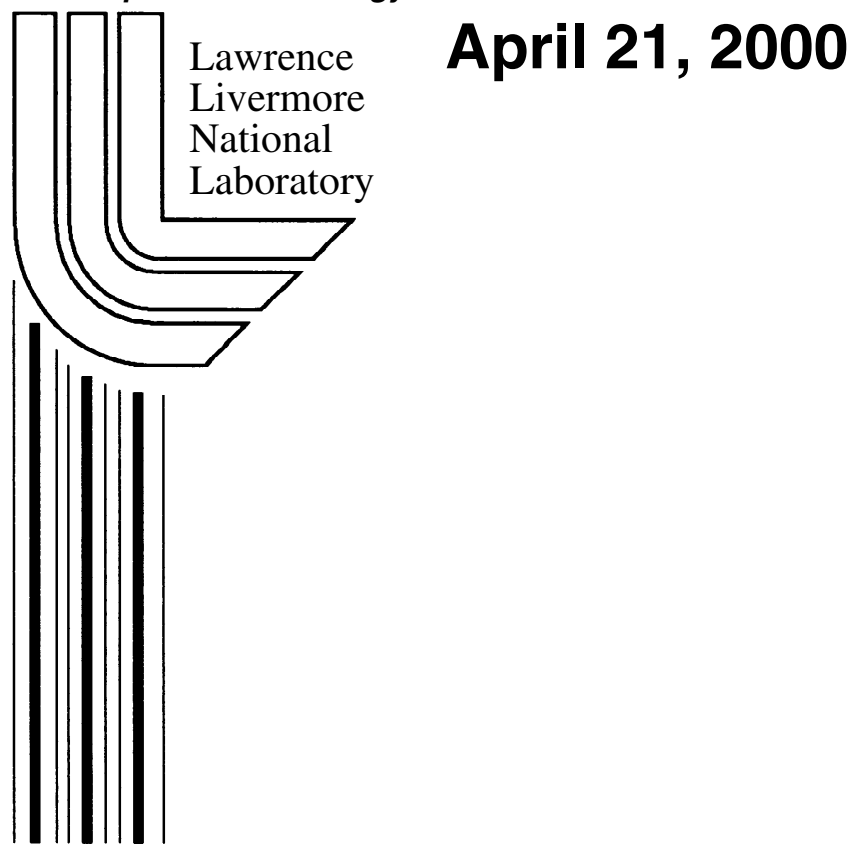




\section{DISCLAIMER}

This document was prepared as an account of work sponsored by an agency of the United States Government. Neither the United States Government nor the University of California nor any of their employees, makes any warranty, express or implied, or assumes any legal liability or responsibility for the accuracy, completeness, or usefulness of any information, apparatus, product, or process disclosed, or represents that its use would not infringe privately owned rights. Reference herein to any specific commercial product, process, or service by trade name, trademark, manufacturer, or otherwise, does not necessarily constitute or imply its endorsement, recommendation, or favoring by the United States Government or the University of California. The views and opinions of authors expressed herein do not necessarily state or reflect those of the United States Government or the University of California, and shall not be used for advertising or product endorsement purposes.

Work performed under the auspices of the U. S. Department of Energy by the University of California Lawrence Livermore National Laboratory under Contract W-7405-Eng-48.

This report has been reproduced directly from the best available copy.

Available to DOE and DOE contractors from the

Office of Scientific and Technical Information

P.O. Box 62, Oak Ridge, TN 37831

Prices available from (423) 576-8401

http://apollo.osti.gov/bridge/

Available to the public from the National Technical Information Service

U.S. Department of Commerce 5285 Port Royal Rd., Springfield, VA 22161 http://www.ntis.gov/

OR Lawrence Livermore National Laboratory Technical Information Department's Digital Library http://www.llnl.gov/tid/Library.html 


\title{
DYNA3D Code Practices and Developments
}

\author{
J. Lin, E. Zywicz, and P. Raboin
}

DYNA3D is an explicit, finite element code developed to solve high rate dynamic simulations for problems of interest to the engineering mechanics community. The DYNA3D code has been under continuous development since 1976[1] by the Methods Development Group in the Mechanical Engineering Department of Lawrence Livermore National Laboratory. The pace of code development activities has substantially increased in the past five years, growing from one to between four and six code developers. This has necessitated the use of software tools such as CVS (Concurrent Versions System) to help manage multiple version updates. While on-line documentation with an Adobe PDF manual helps to communicate software developments, periodically a summary document describing recent changes and improvements in DYNA3D software is needed. The first part of this report describes issues surrounding software versions and source control. The remainder of this report details the major capability improvements since the last publicly released version of DYNA3D in 1996. Not included here are the many hundreds of bug corrections and minor enhancements, nor the development in DYNA3D between the manual release in 1993[2] and the public code release in 1996.

\section{Source Control}

The advent of Laboratory/Industrial partnerships required that software developed under the Cooperative Research And Development Agreement (CRADA) be kept proprietary for a prescribed time period. While versions of DYNA3D software for LLNL users might contain the full capability set, source software shared with other code collaborators could not contain the CRADA developments. To implement this control, UNIX scripts were created to strip proprietary developments from the source code of the publicly-distributed versions. These scripts can build targeted versions of DYNA3D source code for different distributions.

The DYNA3D source code was modified so that protected capabilities are removable in distinct modular subroutines that allow the code to run regardless of the presence of other capabilities. In this way, the primal source code stored in the CVS repository contains all 
code capabilities. The full set of DYNA3D code capabilities includes parallel MessagePassing Interface (MPI) features (ParaDyn), proprietary material models and features developed just for the Department of Defense (DoD), Los Alamos National Laboratory (LANL) and composite material models for the Automotive Composite Consortium CRADA (ACC). None of the above mentioned features are included in the publiclyreleased versions of DYNA3D. The status of distributable features change as capabilities and decisions about source distributions are reviewed.

Creating modular-software developments that are self-contained features is an effective coding practice that has code re-use advantages. Indeed this practice allows software versions to be easily modified with relatively few commands in the compiling makefiles and scripts. The essential feature of this type of development is that the DYNA3D source code uses conditional definition statements "IFDEF'"s that remove all calls to the selectable feature unless that feature has been specifically identified for inclusion. In this way, "backward-engineering" of protected features is impossible, because the code algorithms are removed entirely from the source code. Not even the subroutine calls to these capabilities remain to be deciphered. This is substantially more protective than simply commenting features out.

\section{DYNA3D 2000}

The DYNA3D manual contains descriptions of the basic capabilities up to 1993 and not the full feature set of capabilities mentioned above. For example, the ParaDyn code (parallel DYNA3D) is described in its own manual and is not described in this document. Likewise, the public versions of the DYNA3D code (Collaborator Version) do not contain ParaDyn or any of the capabilities mentioned above. The topics described in the remainder of this document cover the new features being added to the publicly-distributed DYNA3D code. DYNA3D public versions are referred to by the year of their release, so the descriptions herein reference the DYNA3D 2000 version. The list below itemizes the new features and serves as an index for the remainder of this report.

\section{NEW DYNA3D Capabilities}
A. Keyword control entries
B. DYNA3D-NIKE3D link*
C. User termination of dynamic relaxation 
D. Constant time step

E. User defined output file size (unlimited files)

F. Stress update option

G. New rigid wall features

H. New rigid body joints algorithm

I. Flexible spot-weld (Nodal Constraint)

J. Mass proportional damping*

K. Rigid-deformable material switching

L. New options for discrete elements

M. Shell elements with multiple quadrature points

N. New hexahedral element

O. Reaction force (nodal force) output in a text file

P. Maximum material pressure history output

Q. New material models

R. Element deletion

S. New capabilities on contact slide-surfaces

T. DELAM, COHESIVE, and ZIPPER elements

U. SMP multiprocessor threads

V. Mass augmentation

W. MILI database

X. Miscellaneous

*improvement over the existing capabilities

\section{A. Keyword Control Entry}

Free format commands are now used in DYNA3D input decks. In general this capability comes with the following requirements: 1. Place the Keyword commands between the last control card and material entries. 2. Keywords activate features, select options or provide file names. 3. Keyword entries end with an "endfree" card. The set of free format features listed below in Table 1 represent the "new" keywords since 1996. Keywords exist for every control card. For descriptions of these features, refer to the DYNA3D PDF manual. The use of keywords will grow substantially in the future. An interactive edit feature for run-time was added. It allows the user to change select parameters using the keyword format. 
Table 1. Free Format Commands

\begin{tabular}{|c|c|}
\hline Command & Descriptions \\
\hline nikefile file_name & Write a DYNA3D-NIKE3D Link File named file_name. \\
\hline irestt & $\begin{array}{l}\text { Control the continuation of time step and termination time for the } \\
\text { simulation. }\end{array}$ \\
\hline drtime & Dynamic relaxation time limit. \\
\hline drstep & Dynamic relaxation time step limit. \\
\hline normck & Contact segment consistency check for normal orientations. \\
\hline isandb 2 & Debug deleted contact segments with a graphical plot file \\
\hline tslmai $\Delta t$ & Initial mass augmentation time step limit \\
\hline tslmas $\Delta t$ & Steady mass augmentation time step limit \\
\hline ptsming & Fraction of initial time step size for element deletion. \\
\hline tsming & Time step size for element deletion. \\
\hline nfrout & Activate the nodal force output to a text file. \\
\hline matfile file_name & Designate the file that contains the material definitions \\
\hline nodefile file_name & Designate the file that contains the nodal definitions \\
\hline beamfile file_name & Designate the file that contains the beam element definitions. \\
\hline brickfile file_name & Designate the file that contains the hexahedral element definitions. \\
\hline shellfile file_name & Designate the file that contains the shell element definitions. \\
\hline tshellfile file_name & Designate the file that contains the thick shell element definitions. \\
\hline loadcurvefile file_name & Designate the file that contains the load curve definitions. \\
\hline pressurefile file_name & Designate the file that contains the pressure segment definitions. \\
\hline velofile file_name & Designate the file that contains the initial nodal velocity definitions. \\
\hline maxpfile & Activate the maximum material pressure history output. \\
\hline threads $n$ & Run SMP DYNA3D with $\mathrm{n}$ processing threads \\
\hline endfree & End free format commands \\
\hline
\end{tabular}

\section{B. Two-Way DYNA3D-NIKE3D link}


The DYNA3D-NIKE3D link was enhanced with more robust features that make transferring results between the explicit and implicit codes more complete and useful. It is also advantageous to use this feature for DYNA3D to DYNA3D linkages as a simplified and more versatile restart feature. The linkage is made on either the command line ( $m=$ file_name) or with a keyword entry (nikefile). The file includes all nodal, element and slideline information and is valid for selected materials common to both codes. The keyword irestt controls the continuation of simulation time and time step. One of the feature enhancements with this link is the ability to change shell element formulation.

\section{User Termination of Dynamic Relaxation}

When the dynamic relaxation feature of DYNA3D is in effect, it is possible to terminate the relaxation in three different ways. The most direct method is to issue the sense switch command sw5. Then there are two controls via Keyword entries. The Keyword drtime creates a simulation time limit, and the Keyword drstep sets a time step limit.

\section{Constant Time Step}

A constant time step size is activated by using a negative time step factor on control Card 6 . The absolute value will be used as the time step throughout the run. A constant time step size is useful for debugging purposes and if used carefully, it can speed up a simulation. For a restart run, if the new time step factor is less than zero, then the absolute value of the new factor is used as the constant time step. Upon restart if the new time step factor is equal to zero, then the new time step size is inherited from the time step of the previous run. When the restart time step factor is greater than zero, that factor is used as the new time step factor. 


\section{E. User Defined File Size}

Historically, DYNA3D plot files and other binary output were stored in files with a fixed length of 1 Mbyte. This restriction was removed, and file sizes can now be set on the execution command. With $l=f i l e \_s i z e$ included on the DYNA3D execution command, all of the output file families will share the same file size, where file_size is the file size in MB. This capability is only compatible with the GRIZ post processor because TAURUS will only work on files with the default file size (1 MB). While this feature was originally developed to reduce the number of binary files in a file family to fewer than 100, DYNA3D can now generate and GRIZ can read an unlimited number of files in a file family. The maximum length of file names has been increased from 8 to 128 characters.

\section{F. Stress Update Option}

Objectivity for stress integrators can be vitally important to accurate solutions. Specifically, for problems where objects can rotate a few times to thousands of revolutions, the stress fields must be maintained without errors that can grow due to compounded stress update inaccuracies. This topic has been a subject of research [3][4] and DYNA3D implements three formulations of increasing accuracy. The stress update option parameter, iorder, is entered in columns 46-50 on control card 9. With iorder equal to 1, 2 and 3 the update options are the default first-order accurate method, a second-order accurate method and an exponential mapping method, respectively. Higher accuracy comes with a higher cost, but higher accuracy could be very important for problems involving large motions.

\section{G. New Rigid Wall Features}

Two features have been added to the Rigid Wall capabilities. The first is automatic sorting of nodes and the second is frictional contact with rigid walls. To activate the automatic sorting feature, enter zero for the number of rigid wall slave nodes. The purpose of automatic sorting is to have DYNA3D sorts out all nodes between planes passing through point $\mathrm{P}$ and $\mathrm{Q}$ (see Rigid Wall input) and normal to the rigid wall normal vector. To activate frictional contact with a Rigid Wall, enter a negative one for the stick condition flag, ISTICK. A frictional coefficient defines the finite friction between stick and free sliding 
after contact. To complete the frictional Rigid Wall specification, add one additional card after the first Rigid Wall card. This card should include the Friction Coefficient, fric, in E10.3 format in column 1-10.

\section{H. New Rigid Body Joints Algorithm}

The Rigid Body Joints algorithm was replaced with a new algorithm that increases the relative penalty stiffness used in the joint to achieve a tighter bond. The new algorithm eliminates the need to reduce the time step to tighten a rigid body joint. The default penalty stiffness now provides automatic stability.

\section{Flexible Spot-weld (Nodal Constraints)}

The existing Spotweld feature in DYNA3D was modified to allow the distance between two nodes to vary. Add the low and high strain limits immediately after IDOF in $2 E 10.3$ format.

\section{J. Mass Proportional Damping}

New flexibility was added to Mass Proportional Damping by allowing different frequencies to be damped at different times in a simulation. Thus, sets of mass proportional dampening are defined. MDAMP in column 51-55 of control card 7 defines the number of Mass Proportional Damping sets in the model. The individual Mass Proportional Damping sets are placed between One Dimensional Slidelines and Stiffness Proportional Rayleigh Damping.

\section{K. Material Switching Feature}

With material switching it is now possible to change back and forth between deformable and rigid behaviors. The purpose is to save computer time by forgoing the element calculation and/or allowing larger time increments. A rigid material is computationally 
efficient, but should only be used when the model is undergoing negligible deformation. This methodology is useful for multiple-impact problems such as a vehicle hitting multiple highway structures. During impact, the materials should be deformable, but after rebounding and prior to subsequent impact, rigid behavior is optimal for simulating free flight motion. Element stresses/strains are frozen at the time of switching to a rigid phase. Stresses/strains remain unchanged during the rigid phase. This is correct for the shell/beam elements, but not for the continuum elements. A coordinate transformation for continuum element stresses and strains is done at the time of switching back to deformable phase.

\section{New Features for Discrete Elements}

Two new Discrete Elements were added to DYNA3D. The first is a Torsional Spring/Damper and the second is an Orthotropic Spring/Damper.

\section{Fully and Selective-Reduced integrated Shell}

Two additional shell integration methods were added: a fully integrated 4-node shell element and a Selective-Reduced Integrated (SRI) shell element. Both of these formulations are useful in dealing with extreme spatial instabilities.

\section{N. New hexahedral element}

The Mean-Dilatation formulation and fully integrated hexahedral elements are activated by setting the Hourglass control to type $I H Q=6$ and 12 respectively on the Material Control Card. These selections are slower than the under-integrated elements, but they are useful in dealing with extreme spatial instability.

New element formulations based on the variations of physical hourglass control $(I H Q=7,8$, 9 and 10) are now available [5]. The element calculates an approximate enhanced strain field over its domain. With this strain field, the material stiffness and stress at the center of the element, an approximate stress field is constructed to calculate the element forces. The new element is nearly as fast as the ordinary perturbation stiffness hourglass control (IHQ=4 
and 5) but performs as well as the fully integrated incompatible modes elements in many cases. The $I H Q=7,9$ are incremental formulations while $I H Q=8,10$ are the total displacement formulations. The formulation $I H Q=9,10$ use the volume average strain displacement matrix like $I H Q=5$ and therefore they satisfy the patch test. The formulations $I H Q=7,8$ use the element center strain displacement matrix and are slightly faster. Because the hourglass control requires a material stiffness, it has only been implement for materials $1,2,3,18$ and 29.

\section{O. Nodal Forces history output (text file)}

This option provides Nodal Forces history output in a text file. It is activated by the keyword input nfrout. The output nodes are defined in a user-provided node list. The output interval is the same as the time history output. The file name for the output is nodfrc.

\section{P. Maximum Material Pressure history output}

The Maximum Material Pressure history option provides a text output file containing a time history of maximum pressures for a user-defined material list. The option is activated by the keyword option maxpfile. The output interval is the same as the time history output.

\section{Q. New Material Models}

In this release of DYNA3D, there are ten new material models. Several of these models are the contributions of LLNL DYNA3D Collaborators.

1. Power Law Isotropic Elastic-Plastic material for Shell elements (material type 18).

2. Mechanical Threshold Stress (material type 29)

3. Multiple-Material Laminate (material type 42)

4. Transversely Isotropic Visco-Hyperelasticity (material type 43).

5. Rigid Foam (material type 44).

6. New Concrete/Geologic Material [6] (material type 45). 
7. General Anisotropic Elasticity (material type 46)

8. Material Interface Guide (material type 47)

9. Braided Composite Model with Failure (material type 50)

10. Uni-directional Composite Model (material type 56)

The Power Law model (type 18) extends a popular continuum model to shell elements and solves the associated conditions of a plane stress formulation. The Mechanical Threshold Stress model (type 29) is a visco-plastic material model that incorporates temperature and rate effects, and is used primarily to model metals at medium to high loading rates or temperatures. The multiple-material laminate model (type 42) is not truly a constitutive model, but rather a mechanism to use existing shell element models to replicate a "laminate" of dissimilar materials. It is used to model multi-directional composite materials. The Transversely Isotropic Visco-Hyperelasticity model (type 43) models fiber reinforced visco-hyperelastic materials. The Rigid Foam model (type 44) was added to model crushable foams and is useful to modeling helmets in head- impact simulations. The Concrete model (type 45) is a collaborator contribution that has been extremely valuable in simulating the deformation of large structures involving damage and failure. The General Anisotropic Elasticity model (type 46) permits arbitrary elastic symmetry to be modeled. (Only materials with orthotropic or higher symmetry could be modeled before.) The Material Interface Guide (type 47) is a predefined interface to make the addition of externally developed material models into DYNA3D easier. The Braided Composite Model with Failure (type 50) is a progressive damage model for transversely isotropic laminate. It was developed to model the failure of braided composites in automotive structures, and is now available to the general community. Finally, the uni-directional composite model (type 56) simulates the elastoplastic behavior of a uni-directional composite. It permits the detailed response of uni-directional material in micro-mechanical models to be simulated without having to model each fiber and the surrounding matrix.

\section{R. Element Deletion}

In addition to material failure, an element deletion procedure based on a minimum time step size is also available. This permits more realistic simulations and allows element removal to be triggered by multiple mechanisms. Thus, all materials and element types that may become severely distorted and limit the global time step size for the model will be deleted from calculations. The minimum time step size can be defined as an absolute value or a 
fraction of the initial time step. A default global value can be defined and/or individual material number dependent values can be prescribed. This can speed up a simulation and serves to better (robustly) predict failure scenarios. These changes greatly enhance the ability of DYNA3D to model progressive failure with Slidesurfaces with Adaptive New Definition (SAND).

\section{S. New Contact Capabilities}

Advanced contact algorithms are at the heart of a robust and high performance explicit dynamics code. In this vein, developments in the area of contact have improved the accuracy and speed of contact calculations. A free format keyword input section is made available for each individual contact slide-surface. It permits the input of new control data and redefining previously assigned control parameters in a more user friendly and less restrictive manner. Many of the new features and options are activated by the various keyword entries in this section. These keyword entries can also be used to overwrite previously defined contact parameters or options.

Two new capabilities give better diagnostics to debug contact geometry. The first is a contact segment consistency check on slide surface normals. Activated by the keyword entry normck, contact segments that are mis-orientated with respect to each other are identified for correction by the user. The second new diagnostic is for automatic contact. With the keyword $i s a n d b$ 2, DYNA3D makes a diagnostic calculation of detected contact conditions, and then writes a plot database of possible contact surface penetrations/violations. With this information, a user can fix the model geometry to properly pose the contact conditions. Both normck and isandb apply to all contact slidelines, thus must be part of the general keyword input section between the last control card and the beginning of the material input. An additional parameter associated with a specific slideline that provides some initial surface detection flexibility is a dimensional tolerance $g$. This is activated with the keyword entry pen_thres $g$ in the slideline keyword section.

For all slide surface types, segments associated with shell elements are automatically removed when the associated shell element fails. 
A new slide-surface idealization was developed. It allows a contacting object to be idealized as a portion of an analytical surface - specifically a sphere. This feature works with type 3 contact and alleviates discretization issues.

A feature to permit initial slide surface inter-penetrations was added. It allows the user to scale, with time, the amount of perceived inter-penetration via a load curve. Also, select slide surfaces may be activated and deactivated during an analysis at specified times.

Lagrange contact modeling [7] is an add-on contact capability for slide-surface types 3, 5, $10,12,13$ and 14. It offers several advantages over traditional penalty based algorithms. The force generating algorithm poses no time-step size limitations, and the solution contains no inter-penetration. There is no dependency on contact scale factors or material stiffness. Another advantage is that elastic contacts can be generated between rigid bodies. Lagrange contact applications include high velocity impacts, contacts between very dissimilar materials, e.g. foam and steel, and high fidelity rigid body interactions. In testing, the Lagrangian solver (a preconditioned conjugate-gradient projection method) adds between 3 and $15 \%$ to the total computational cost. Lagrange contact is activated by keyword entry lagop in the slideline keyword section.

A self-generation feature for traditional contact surfaces has been added. It allows the master and slave surfaces (or nodes) to be specified via material numbers and, optionally, several geometric factors. This feature removes the burden of explicitly defining the necessary segments in the same manner as automatic contact (types 12, 13, and 14).

Automatic contact's underlying sorting and searching algorithms, used by types 12,13 , and 14 , were entirely rewritten. The new approach permits nodes to be in contact with multiple segments. Thus, with the changes made in the basic algorithm, both the robustness and computational speed of automatic contact is greatly improved.

Automatic and SAND contact were capabilities first released in the 1996 version of DYNA3D. During the interim, the speed and robustness of these algorithms were improved and combined into type 14 slide-surfaces. The new automatic contact algorithm is now also used by the SAND contact routines. The new version permits arbitrary contact between all active segments (unlike type 11), it appreciably reduces user input and it is fully compatible with the Lagrangian contact solver. 


\section{T. DELAM, COHESIVE, and ZIPPER Elements}

Three types of 2-dimensional elements were added - DELAM, COHESIVE, and ZIPPER elements. These elements use a traction-displacement law to generate forces based upon the displacement jump across the 2-D surface. DELAM, COHESIVE, and ZIPPER elements are used between shell element faces, brick element faces, and shell element ends (a 1-D representation of a 2-D surface), respectively, to model separation or fracture. Several traction-displacement constitutive laws were developed for each.

\section{U. SMP Multiprocessor Threads}

DYNA3D can utilize shared-memory parallelism hardware architectures available on highend SGI, SUN, DEC and IBM workstations with software threads (OpenMP). Using this feature is extremely easy: use a Keyword entry threads $n$, where $n$ is the number of threaded processors. With many caveats pertaining to problem size and other particulars, performance testing shows sub-linear scaling speedups with the performance saturating at about 8 processors. This is sure to change in the future.

\section{Mass Augmentation}

A new feature, called mass augmentation, was incorporated. This option adds mass to select elements in order to maintain a critical time-step size above a prescribed threshold value. This permits the use of a larger time step. The keyword entries tslmai $\Delta t$ and tslmas $\Delta t$ set the desired time step size for DYNA3D at initialization and for steady conditions, respectively. The feature, originally developed and contributed by a collaborator, has been extensively debugged and is available for brick, beam, and shell elements. Judicious use of mass augmentation can dramatically reduce the execution time to solve DYNA3D analyses.

\section{W. MILI Plot Database}

The TAURUS database that has stood in good service for 25 years was replaced with a new database called MILI (Mesh I/O Library). MILI is a "self-describing database with highlevel, finite element analysis-cognizant semantics, and cross-platform data portability." On 
a practical level, DYNA3D with MILI provides a forward path so that any or all of the modeling parameters and results can be displayed. Initially, MILI replaces TAURUS with few noticeable improvements (global variables), but this is expected to change soon.

\section{Miscellaneous}

The YASE element was extended to correctly treat orthotropic materials.

An implicit integration operator was added for rigid bodies. The new operator exactly preserves momentum and energy, even for the rotational part.

A new load curve type was formed. It permits independent dynamic relaxation and transient profiles to be specified in one load curve.

The input format for "Material initialization for rotational motion" and for "Body force loads by material" options were generalized. They now permit each part in an analysis to have its set of initial conditions or body forces.

\section{Future Work}

Many features under development are not yet ready for public release. These features may be ready in the near future so they are included here to indicate our current directions for code development.

- Non-iterative elastic-plastic constitutive models for shell and continuum elements.

- New shell elements capable of handling extreme structure geometry.

- Multiple time step integration.

- User manual update and release 


\section{References}

[1] Hallquist, J.O., "Preliminary User's Manuals for DYNA3D and DYNAP (Nonlinear Dynamic Analysis of Solids in Three Dimension), " University of California, Lawrence Livermore National Laboratory, (1976) and Rev. 1 (1979), Report UCID-17268.

[2] Whirley, R.G., and Engelmann, B.E., "DYNA3D: A Nonlinear, Explicit, ThreeDimensional Finite Element Code for Solid and Structural Mechanics - User Manual", University of California, Lawrence Livermore National Laboratory (1993), UCRL-MA107254.

[3] Simo and Vu-Quoc, "On the Dynamics in Space of Rods Undergoing Large Motions A Geometrically Exact Approach", Computer Methods in Applied Mechanics and Engineering, 66, (1988),125-161.

[4] Simo and Vu-Quoc, "Unconditionally Stable Algorithms for Rigid Body Dynamics That Exactly Preserve Energy and Momentum", International Journal for Numerical Methods in Engineering, 31, (1991), 19-52.

[5] Puso, M.A., "A Highly Efficient enhanced assumed strain physically stabilized hexahedral element", International Journal for Numerical Methods in Engineering, (in press).

[6] Malvar, L.J., Crawford, J.E., Wesevich, J.W., and Simons, D., "A New Concrete Material Model for DYNA3D, Release II: Shear Dilation and Directional Rate Enhancement", A Report to Defense Nuclear Agency under Contract No. DNA001-91C-0059 (1996).

[7] Zywicz, E., and Puso, M.A., “A General Conjugate-Gradient Based Predictor-Corrector Solver for Explicit Finite-Element Contact," International Journal for Numerical Methods in Engineering, (1999), 439-459. 